\title{
Falhas de mercado e redes em políticas públicas: desafios e possibilidades ao Sistema Único de Saúde
}

\author{
Market and public policy network failures: challenges \\ and possibilities for the Brazilian Unified Health System
}

Francisco Percival Pinheiro Filho ${ }^{1}$

Flávia Mori Sarti ${ }^{1}$

${ }^{1}$ Programa de Pós-

Graduação em Modelagem de Sistemas Complexos,

Escola de Artes, Ciências e

Humanidades, Universidade de São Paulo (EACH-USP). Av. Arlindo Béttio 1000,

Ermelino Matarazzo.

03828-000 São Paulo SP.

francisco.pinheiro@usp.br

\begin{abstract}
The principles and guidelines of the Brazilian Unified Health System (SUS) impose a healthcare service structure based on public policy networks which, combined with the financing model adopted, leads to market failings. This imposes barriers to the management of the public health system and the enactment of SUS objectives. The institutional characteristics and the heterogeneity of players, allied to the existence of different healthcare approaches, generate analytical complexity in the study of the global dynamics of the SUS network. There are limitations in the use of quantitative methods based on static analysis of retrospective SUS data. Thus, an approach taking SUS as a complex system using innovative quantitative methodology based on computational simulation is proposed. This paper sought to analyze challenges and possibilities of the combined application of cellular automata modeling and agent-based modeling for simulation of the evolution of the SUS healthcare service network. This approach should permit better understanding of the organization, heterogeneity and structural dynamics of the SUS service network and a minimization of the effects of market failings on the Brazilian health system.
\end{abstract}

Key words Healthcare management, Market failings, Brazilian Unified Health System, Public policy networks, Complex system, Agent-based modeling
Resumo Os princípios e as diretrizes do Sistema Único de Saúde (SUS) impõem uma estrutura de assistência baseada em redes de políticas públicas que, combinada ao modelo de financiamento adotado, conduz a falhas de mercado. Isso impõe barreiras à gestão do sistema público de saúde e à concretização dos objetivos do SUS. As características institucionais e a heterogeneidade dos atores, aliadas à existência de diferentes redes de atenção à saúde, geram complexidade analítica no estudo da dinâmica global da rede do SUS. Há limitações ao emprego de métodos quantitativos baseados em análise estática com dados retrospectivos do sistema público de saúde. Assim, propõe-se a abordagem do SUS como sistema complexo, a partir da utilização de metodologia quantitativa inovadora baseada em simulação computacional. O presente artigo buscou analisar desafios e potencialidades na utilização de modelagem com autômatos celulares combinada com modelagem baseada em agentes para simulação da evolução da rede de serviços do SUS. Tal abordagem deve permitir melhor compreensão da organização, heterogeneidade e dinâmica estrutural da rede de serviços do SUS e possibilitar minimização dos efeitos das falhas de mercado no sistema de saúde brasileiro.

Palavras-chave Gestão em saúde, Falhas de mercado, Sistema Único de Saúde, Redes de políticas públicas, Sistema complexo, Modelagem baseada em agentes 


\section{Introdução}

A investigação de falhas dos mecanismos de mercado no setor da saúde constitui-se como linha de pesquisa prolífica desde a década de 1960, a partir do trabalho seminal de Kenneth Arrow. ${ }^{1}$ Particularmente, destacam-se características diferenciadas associadas à natureza da demanda, às condições de oferta, à incerteza quanto à necessidade e à provisão dos bens e serviços de saúde, ao estabelecimento de preços e ao comportamento dos agentes envolvidos ${ }^{1}$.

De fato, ações em saúde podem ser insuficientes (como programas de controle de vetores), custosas demais (como saneamento básico), ou virtualmente inexequíveis (como segurança do alimento e sistemas de informação em saúde) sob a ótica do setor privado ${ }^{2,3}$.

As denominadas falhas de mercado resultam em diversos problemas na operacionalização dos sistemas de saúde nacionais, a partir da discussão quanto ao grau de atuação do governo na provisão de assistência em saúde ${ }^{3,4}$.

Consequentemente, a gestão da saúde difere marcantemente entre países, incluindo desde modelos de sistemas de saúde integralmente públicos até modelos predominantemente privados, nos quais somente ações ligadas à saúde pública, restritas ao sistema de vigilância sanitária e epidemiológica, são assumidas pelo governo ${ }^{3,4}$.

No caso brasileiro, especificamente, o delineamento das diretrizes gerais do sistema de saúde baseou-se na busca pela garantia de promoção, proteção e manutenção da saúde da população, assim como prevenção, tratamento e reabilitação de doenças ou agravos, especialmente no que tange à definição do papel do setor público em saúde. Desde a inclusão do capítulo da saúde na Constituição Federal em 1988, as diretrizes das políticas públicas incluem descentralização, universalidade, integralidade, equidade e controle social $^{5}$.

Em termos administrativos, a organização do sistema público de saúde deve seguir três diretrizes operacionais ${ }^{6}$ :

1. A descentralização das políticas deve apresentar direção única em cada esfera de governo, com ênfase na prestação dos serviços pelos municípios;

2. A regionalização da rede de serviços deve buscar promover resolutividade e eficiência na provisão de assistência; e,

3. A hierarquização da infraestrutura de serviços em diferentes níveis de complexidade deve congregar participação dos municípios em redes de políticas de saúde.
No entanto, o processo de descentralização das políticas sociais no Brasil apresenta significativas barreiras: características da estrutura federativa brasileira, desigualdades regionais expressivas e ausência de infraestrutura fiscal e administrativa que tendem a dificultar a implementação da descentralização de forma efetiva ${ }^{7}$.

O resultado da descentralização das políticas de saúde torna-se fortemente dependente de três grupos de fatores. Em um primeiro grupo reúnem-se os atributos institucionais das políticas: regras constitucionais, requisitos de engenharia operacional e legado das políticas prévias. O segundo grupo de fatores inclui atributos estruturais das unidades locais de governo, a partir de suas capacidades econômica, fiscal e administrativa. Por fim, o terceiro grupo engloba fatores relacionados à ação política: relações entre Estado e sociedade e intergovernamentais ${ }^{7}$.

A partir da Constituição Federal de 1988, que definiu as bases para a implantação do Sistema Único de Saúde (SUS), reconhece-se a saúde como um direito social da população e um dever do Estado. Portanto, os princípios do Sistema Único de Saúde estipulam a necessidade de um conjunto de políticas sociais e econômicas que devem buscar redução do risco de doença e garantia do acesso universal e integral às ações e serviços de saúde no país, a partir de igualdade de condições e segundo suas necessidades, sob um modelo de financiamento que combina subsídio à demanda na atenção primária por meio de remuneração per capita e o subsídio à oferta em serviços de média e alta complexidade via remuneração por produção $0^{6}$.

Considerando o conceito amplo de saúde disseminado pela Organização Mundial da Saúde ${ }^{8}$ de estado de pleno bem-estar físico, mental e social, as políticas públicas de saúde devem contemplar ações interssetoriais de considerável amplitude e magnitude para promoção da saúde da população.

A interssetorialidade intrínseca às políticas públicas de saúde é reforçada pela verificação de inúmeras externalidades positivas e negativas que impactam sobre a saúde da população, a partir de ações em diferentes setores de atividade ${ }^{2,4}$.

A partir dos inúmeros aspectos elencados, verifica-se que a gestão de sistemas de saúde inicia-se pela coordenação de atores que apresentam certo grau de independência decisória entre si e, ao mesmo tempo, influenciam-se mutuamente na adoção de estratégias para desenvolvimento de suas atividades.

Tais características imprimem um caráter de complexidade à saúde: los sistemas de servicios de 
salud reúnen las características de un SCA [sistema complexo adaptativo]: sensibilidad a sus condiciones iniciales, autoorganización, propiedades emergentes y autosimilitud ${ }^{9}$.

Assim, a gestão de sistemas de saúde deveria ser conduzida sob o paradigma de análise dos sistemas complexos, uma área de conhecimento inovadora baseada em metodologia de investigação quantitativa que reconhece a importância da atuação individual dos agentes e, simultaneamente, sua influência sobre o comportamento global do sistema complexo que compõem.

O objetivo do presente artigo é analisar potencialidades da aplicação da metodologia à gestão do sistema de saúde brasileiro, via construção de redes em políticas públicas de saúde e simulação baseada em agentes para redução dos percalços causados pelas falhas de mercado presentes no sistema público de saúde brasileiro.

\section{Falhas de mercado no sistema de saúde brasileiro}

A atuação do governo no setor de saúde torna-se necessária para direcionamento, correção ou complementação das atividades do setor privado, face à existência das falhas de mercado. Tal atuação pode ocorrer via intervenção direta (como, por exemplo, a produção de bens públicos) ou ação indireta (regulação dos mercados) ${ }^{10}$.

As características diferenciadas da demanda e oferta no setor de atenção à saúde, descritas por Arrow $^{1}$, são particularmente sensíveis à imprevisibilidade na ocorrência de doenças ou agravos, que geram incertezas quanto à necessidade de serviços de saúde pelos indivíduos na sociedade.

Musgrove $^{3}$ delimita três domínios da saúde, a partir do custo e natureza pública ou privada da provisão de bens, incluindo os respectivos argumentos à intervenção governamental:

1. Bens e serviços públicos ou meritórios, cuja produção no âmbito do setor privado situa-se aquém das necessidades em saúde da população, sendo papel do governo assegurar a produção em nível ótimo;

2. Bens e serviços de saúde de custo catastrófico, cuja magnitude orçamentária ultrapassa a capacidade de pagamento dos indivíduos, sendo papel do governo subsidiar o atendimento à população;

3. Intervenções privadas de baixo custo em saúde, sendo papel do governo garantir a eficiência e a equidade em termos de provisão de assistência à saúde, seja pelo setor privado ou público.

A existência de falhas de mercado exige ação compulsória do governo na provisão de assis- tência à saúde nos dois primeiros domínios delimitados $^{3,4,11}$, sendo opcional a atuação do setor público somente no terceiro caso.

Além das falhas de mercado mencionadas, o setor de saúde, devido às incertezas inerentes ao processo saúde-doença, também apresenta assimetria de informação entre agentes participantes do sistema: pacientes, profissionais de saúde, estabelecimentos provedores de bens de saúde e governo. A assimetria informacional reflete a indisponibilidade de informação relevante ao estabelecimento de relações entre agentes, a partir de decisões suficientemente informadas ${ }^{11,12}$.

\section{Infraestrutura do Sistema Único de Saúde: falhas de monopólio natural}

A análise da estrutura da rede de serviços do SUS deve ser considerada a partir da relação entre a demanda e a oferta de serviços de saúde sob condições de falhas nos mecanismos de mercado.

Assim, a construção de um sistema de saúde a partir do pressuposto de acesso universal da população aos diferentes níveis de complexidade de ações em saúde, segundo sua necessidade individual, requer estabelecimento de uma rede de serviços com elevada capacidade de atendimento, disponibilidade de atuação e escopo de ação, que busque constantemente incorporar novas tecnologias relativas a produtos e serviços terapêuticos e diagnósticos.

Ademais, tendo em vista as significativas diferenças na rede de prestação de serviços ao SUS, que inclui prestadores de serviço de saúde públicos e privados, há dificuldades substanciais na determinação dos custos das ações em saúde.

Em outras palavras, o cumprimento dos princípios do SUS requer uma rede de prestação de serviços cujo financiamento é caracterizado por elevado custo fixo e baixo custo marginal, uma situação que qualifica existência de um monopólio natural.

As duas soluções plausíveis de atuação do governo em setores sob monopólio natural referem-se à regulação ou à produção pelo setor público $^{10}$.

No primeiro caso, a regulação pressupõe a existência de uma agência reguladora que analise a oferta do serviço de saúde, de forma a estipular o custo médio de produção, base para cálculo do preço final ao usuário ${ }^{10}$.

A solução de regulação pelo setor público conduz, necessariamente, à discussão da viabilidade de efetuar estimativa do custo médio dos serviços de saúde em cada um dos estabeleci- 
mentos de assistência à saúde sob o SUS. Ademais, há necessidade de adoção de um modelo de remuneração suficiente à manutenção dos estabelecimentos prestadores de serviços de saúde, tendo em vista os custos incorridos.

De qualquer forma, a regulação é inadequada à medida que, por um lado, exclui qualquer incentivo ao controle de custos por parte dos prestadores de serviço de saúde e, por outro lado, inviabiliza o processo orçamentário para determinação do volume de recursos necessários ao financiamento do SUS.

No segundo caso, a produção do bem ou serviço pelo setor público requer subsídio parcial ou completo da oferta de assistência à saúde ${ }^{10}$. A hipótese de produção pelo governo assemelhase, à primeira vista, ao modelo atualmente adotado no âmbito do SUS.

No entanto, a viabilidade de aferição do custo médio dos serviços de saúde prestados em cada um dos estabelecimentos do SUS também constitui uma questão presente no modelo de produção pública, tendo em vista a necessidade de estabelecer o valor do subsídio.

Considerando-se a gratuidade na provisão dos serviços de saúde pelo SUS, ainda existe o mesmo problema identificado no caso da regulação: há incentivos adversos no controle de custos aos prestadores de serviços e dificuldade no estabelecimento do volume de recursos necessários ao financiamento do SUS.

\section{Financiamento do Sistema Único de Saúde: entre ineficiência e seleção adversa}

Em última instância, a partir da ótica de um setor de atividade sob monopólio natural, é possível questionar a perenidade do sistema de saúde em sua conformação atual, tendo em vista a combinação de prestação de serviços em diferentes estruturas produtivas (estabelecimentos de saúde) com um modelo de financiamento misto, que estipula valores fixos de remuneração per capita ou por produção de procedimento específico, em função da complexidade do serviço prestado.

A partir das diferenças de estrutura entre estabelecimentos prestadores de serviços de saúde do SUS, há substanciais diferenças de custo à oferta de assistência à saúde. No modelo de financiamento atualmente vigente no âmbito do SUS, há três situações possíveis:

1. Caso o valor da remuneração pelo SUS seja equivalente ao maior custo para prestação do serviço de saúde verificado entre estabeleci- mentos de saúde, as demais unidades auferem lucro econômico;

2. Caso o valor da remuneração pelo SUS seja equivalente ao menor custo para prestação do serviço de saúde verificado entre estabelecimentos de saúde, as unidades com custo superior têm prejuízo, implicando em suspensão da prestação de serviços contratada;

3. Na hipótese do valor da remuneração pelo SUS ser equivalente ao custo médio prestação do serviço de saúde verificado entre estabelecimentos de saúde, parte das unidades aufere lucro econômico e parte apresenta prejuízo.

Assim, além da dificuldade em determinar o custo médio referente à prestação de cada tipo de serviço de saúde, a escolha do modelo de remuneração da assistência à saúde no âmbito do SUS pode conduzir a paradoxos de seleção dos estabelecimentos de saúde para prestação de serviços: no primeiro caso, há persistência de um padrão de assistência à saúde baseado em ineficiência produtiva (dado que qualquer estabelecimento de saúde aufere lucro econômico); no segundo caso, há geração de monopólio na oferta de serviços de saúde pelo setor público (dado que qualquer estabelecimento de saúde apresenta prejuízo); e, no terceiro caso, há escassez na oferta de assistência à saúde no âmbito do SUS (dado que significativa parcela dos estabelecimentos de saúde é excluída do sistema público).

\section{Organização do Sistema Único de Saúde: deseconomias de escala}

A descentralização e a regionalização da rede de serviços do SUS sob responsabilidade da União, Distrito Federal, estados e municípios resulta em uma organização baseada em sub-redes do sistema público de saúde em cada esfera de governo. Cada rede local é integrada por uma básica de assistência à saúde (atenção básica), serviços classificados nos grupos de procedimentos de média complexidade e serviços especializados de alta complexidade ${ }^{6}$.

Em última instância, o número de redes de prestação de serviços de saúde no âmbito do SUS poderia ser equivalente ao número de entes da federação. No entanto, em função do porte e da demanda de serviços de saúde em cada município, a atenção de média e alta complexidade é organizada regionalmente ${ }^{6}$.

A partir de um sistema de saúde com elevado custo fixo, conforme anteriormente descrito, a dispersão da oferta de serviços de saúde em diversas sub-redes de estabelecimentos no SUS caracteriza 
uma situação de deseconomia de escala, que implica em maior dificuldade na determinação de um modelo de financiamento estável ao SUS ${ }^{13}$.

O sistema de financiamento misto do SUS, aliado à autonomia de gestão de cada esfera de governo, potencializa a expansão da rede de atendimento de média e alta complexidade, gerando elevação dos custos fixos do sistema de saúde e, ao mesmo tempo, promovendo maior fragmentação da oferta de assistência à saúde entre diversos estabelecimentos prestadores de serviço ${ }^{13}$.

Consequentemente, há um ciclo de aprofundamento da deseconomia de escala do sistema de saúde brasileiro pela elevação do custo unitário dos procedimentos e acirramento da disputa intergovernamental pelos recursos financeiros disponíveis, distribuídos no processo orçamentário.

\section{Gestão do Sistema Único de Saúde: relação agente-principal}

A gestão da rede de serviços de saúde do SUS, a partir de suas características de descentralização com direção única em cada esfera de governo e seu modelo de financiamento misto, também caracteriza uma relação principal-agente.

Uma parcela das atribuições do SUS é exercida de maneira comum pela União, estados, Distrito Federal e municípios, enquanto outra parcela das atribuições é específica a cada uma das diferentes esferas de governo ${ }^{6}$.

O modelo de financiamento do SUS prevê a transferência regular de recursos da União para Distrito Federal, estados e municípios, assim como transferência regular de recursos dos estados para municípios ${ }^{6}$.

Parte dos recursos financeiros, relativos à remuneração per capita, são transferidos automaticamente às unidades subnacionais de governo antes da execução das ações e serviços de saúde programados. As informações sobre condições de prestação de serviços de saúde nas redes locais, volume de serviços efetivamente prestados e resultados alcançados estão sob controle dos gestores das redes de serviços de saúde e estabelecimentos prestadores de serviços ${ }^{6}$.

É possível identificar as cinco características da relação agente-principal na situação descrita ${ }^{14}$ :

1. Delegação de tarefas entre esferas de governo, assim como entre governo e estabelecimentos prestadores de serviço de saúde, a partir de condiçõos preestabelecidas;

2. Assimetria de informação dos gestores públicos entre si e em relação aos estabelecimentos prestadores de serviço de saúde;
3. Dificuldade em estabelecer uma relação direta entre transferência de recursos financeiros e esforço empreendido pelos estabelecimentos responsáveis na prestação dos serviços de saúde;

4. Alto custo de monitoramento governamental em relação às ações de saúde realizadas pelos estabelecimentos prestadores de serviço;

5. Falhas no alinhamento dos objetivos entre esferas de governo, dada sua autonomia no âmbito da gestão do SUS.

A remuneração por volume de produção de serviços, no caso dos procedimentos de média e alta complexidade, também reforça as dificuldades na oferta de atenção à saúde, pois facilita o surgimento de risco moral na relação entre usuários e estabelecimentos prestadores de serviços de saúde. A assimetria de informação entre ambos possibilita indução da demanda, ou seja, tendo em vista o conhecimento especializado envolvido em decisões de saúde, é possível induzir o usuário ao uso de serviços de saúde adicionais, mesmo desnecessariamente, de forma a elevar a remuneração obtida pelo estabelecimento prestador de serviços ${ }^{15,16}$.

A situação de risco moral implica, em curto prazo, na expansão desnecessária da oferta de serviços de saúde de média e alta complexidade e, em longo prazo, no aprofundamento da situação de fragmentação da oferta de serviços de saúde, deseconomia de escala, elevação do custo unitário dos procedimentos e acirramento da disputa pelos recursos financeiros disponíveis.

\section{Redes de políticas públicas no âmbito do SUS}

A estrutura da oferta de assistência à saúde representada pela rede do Sistema Único de Saúde pode ser apreendida a partir dos vários arranjos institucionais estipulados à sua estrutura, financiamento, organização e gestão.

As redes de atenção à saúde têm como objetivo garantir a integralidade da atenção à saúde, a partir de integração logística e gerenciamento de pontos de atenção e pontos de apoio diagnóstico com diferentes níveis de complexidade distribuídos em um território determinado. Assim, a política pública de saúde brasileira é estruturada em redes de atenção à saúde que têm capacidade de explicitar diversas modalidades de assistência ${ }^{13}$.

Além das dimensões anteriormente definidas, ainda há ações em saúde organizadas em níveis de complexidade e em áreas temáticas, a partir de necessidades específicas. 
A hierarquização segundo nível de complexidade dos serviços de saúde prestados é definida em função dos requerimentos tecnológicos e especialização dos profissionais de saúde envolvidos, distribuindo-se em redes hierarquizadas de assistência à saúde de nível primário, secundário e terciário.

As redes temáticas de saúde são estruturadas em torno de um agravo, patologia ou fase do ciclo de vida de uma determinada população, comportando serviços de diferentes níveis de complexidade.

Segundo Teixeira e Ouverney ${ }^{17}$, redes de políticas públicas policêntricas e reticulares, à semelhança do SUS, constituem fenômeno recente, sendo caracterizadas por:

- Envolvimento de múltiplos atores em diversas etapas do ciclo das políticas públicas;

- Dependência mútua entre atores componentes da rede;

- Vínculos estabelecidos pela necessidade de instauração de objetivos comuns;

- Difusão e dispersão do controle de recursos e processos; e,

- Dinâmica gerencial intrínseca.

As redes de políticas públicas, embora sejam adequadas à solução de problemas complexos, apresentam uma série de desafios administrativos no que tange aos processos decisórios, ao planejamento e à avaliação, impondo restrições à sua criação, articulação e manutenção ${ }^{17}$.

Misoczky ${ }^{18}$ define redes de políticas públicas como construções alternativas às estruturas hierarquizadas, cuja capacidade para circunscrever processos envolvendo múltiplos agentes e conexões permite vinculação a um território e aos atores. O sucesso das redes de políticas públicas depende de aspectos políticos e administrativos, atores e recursos envolvidos, qualidade e custo das interações estabelecidas.

Apesar da posição crítica de Misoczky ${ }^{18}$ à denominada "hierarquia dos lugares", destacam-se quatro componentes nas formas de apreensão da rede de serviços de assistência à saúde do SUS: envolvimento de múltiplos atores, estabelecimento de conexões entre agentes, vinculação a um território e regras de operacionalização instituídas.

A combinação dos quatro componentes da rede de serviços do SUS perpassa os desafios postos pela gestão de redes de políticas públicas, sugerindo que o Sistema Único de Saúde apresenta-se em permanente processo de construção. O resultado do processo depende da interação estabelecida entre agentes, a partir das condições estipuladas, com vistas à superação dos desafios de gestão existentes.
Assim, a cada nova configuração do sistema público de saúde brasileiro, emergem interações constituídas com objetivo de enfrentar um novo grupo de desafios. Após a superação de um conjunto de desafios, é necessária uma nova rodada de interações, que configuram um novo SUS. O entendimento das características de cada uma das partes componentes da rede do SUS não permite explicar o conjunto, na medida em que o sistema público de saúde é resultado da interação de suas partes; renovando-se ciclicamente em um processo dinâmico, adaptativo e transformador a cada iteração.

Consequentemente, a rede do sistema público de saúde brasileiro deve ser analisada como um sistema complexo adaptativo, isto é, um sistema que assume diferentes configurações e reage aos estímulos do ambiente em que se insere ${ }^{19}$.

\section{Desafios e possibilidades na modelagem do SUS como sistema complexo}

Em geral, a pesquisa quantitativa no campo da saúde é baseada em análises estatísticas usualmente conduzidas na forma de correlações, regressões e modelos estruturais - a partir de dados retrospectivos, provenientes de pesquisas populacionais ou intervenções, buscando-se projetar resultados futuros dos efeitos identificados no passado recente, construindo-se estudos preditivos com alto grau de incerteza.

A abordagem do Sistema Único de Saúde como um sistema complexo adaptativo possibilita a utilização de ferramentas de modelagem e simulação que, por um lado, facilitam a compreensão de sua dinâmica estrutural e, por outro, permitem analisar seu potencial a partir dos padrões estabelecidos às interações entre seus agentes e, portanto, sua configuração em rede de políticas públicas de saúde.

A rede de prestação de serviços do SUS apresenta como premissas básicas: descentralização em cada unidade da federação, hierarquização em níveis de complexidade da atenção à saúde, regionalização em sub-redes institucionais para atendimento da demanda em saúde populacional com eficiência e modelo de financiamento misto, a partir de regras estritas para articulação dos múltiplos atores envolvidos, resultando em incentivos ao estabelecimento de relações entre esses atores.

A incerteza decorrente da imprevisibilidade na ocorrência de doenças ou agravos e as falhas de mecanismos de mercado no setor da saúde ${ }^{1}$, aliadas à complexidade da rede social de atores 
que participam do SUS, são características que permitem a proposta de análise do sistema público de saúde brasileiro na forma de um sistema complexo, a partir da aplicação de modelagem com autômatos celulares (AC) e modelagem baseada em agentes (MBA).

A modelagem por autômatos celulares e a modelagem baseada em agentes, por sua vez, permitem uma abordagem quantitativa prospectiva, a partir do uso de programação computacional para formulação de vários cenários possíveis de interação entre atores sociais na forma de uma simulação computacional baseada em parâmetros próximos à realidade. $\mathrm{O}$ modelo de simulação computacional apresenta retroalimentação no comportamento dos atores sociais, sendo passível de reprodução, teste e análise estatística dos cenários resultantes de várias rodadas de interação entre atores sociais, realizadas de forma semelhante à projeção de uma realidade virtual.

A modelagem por autômatos celulares (AC) refere-se à representação do sistema de relações entre atores - rede social - em formato semelhante a um tabuleiro de xadrez, onde os atores constituem células espacialmente localizadas com regras simples de relacionamento ou interação com outras células vizinhas. A estrutura da rede social representada pelas interações entre os atores ou células evolui ao longo do tempo em função da situação inicial dos atores, da sua vizinhança e da regra de interação - normas de relacionamento entre atores -, determinando a estrutura global, comportamentos e propriedades da rede social em estudo ${ }^{19}$.

A modelagem com autômatos celulares permite o trabalho com diferentes tipos de atores (células com diferentes propriedades), possibilitando a construção de um modelo cujos atores sociais apresentem características de comportamento individual e de interação social semelhantes às relações identificadas entre os atores do Sistema Único de Saúde, por exemplo.

A modelagem baseada em agentes (MBA) é realizada a partir da caracterização dos atores que participam de uma rede social de formato pré-definido. Os agentes ou grupos de agentes da MBA têm regras de comportamento simples, definidas a partir dos incentivos (payoffs) que obtêm na rede de interações sociais. Os processos de tomada de decisão de cada agente são autônomos, no entanto, apresentam um certo grau de interdependência (devido à resposta dos demais agentes em relação à decisão tomada pelo indivíduo), adaptação (devido à existência de reação aos estímulos externos captados pelo indi- víduo) e responsividade (devido aos efeitos de memória quanto às ações passadas e às reações dos demais agentes). Ou seja, o comportamento dos agentes é contingencial, dependente do nível de acesso às informações do restante do sistema no qual estão inseridos ${ }^{19}$. O sistema complexo evolui a partir do comportamento e das interações entre agentes, sendo possível identificar a formação de estruturas organizacionais e padrões de comportamento ${ }^{20}$. A complexidade do sistema é resultado direto das interações entre agentes (ação individual - resposta social - reação individual), não de sua complexidade cognitiva.

O uso de modelagem com autômatos celulares $(A C)$ e modelagem baseada em agentes (MBA) permite o estudo de sistemas marcados pela existência de agentes e espaços múltiplos e heterogêneos, cujas relações e resultados não são lineares, sendo necessárias ferramentas de análise dinâmica e espacial ${ }^{21}$.

É possível ao pesquisador, por exemplo, gerar um modelo computacional de sistema de saúde hipotético, cujos parâmetros de operacionalização sejam idênticos aos princípios e diretrizes do Sistema Único de Saúde. Isso permite iniciar uma simulação que reproduz as condições atuais do sistema público de saúde brasileiro e, em seguida, possibilita ao pesquisador modificar incentivos no financiamento e formas de gestão do sistema de saúde virtual, de forma a analisar a resposta obtida no comportamento dos atores sociais virtuais.

Algumas questões atualmente pendentes no estudo da rede de serviços do SUS poderiam obter melhores resultados pela aplicação de modelagem com autômatos celulares e modelagem baseada em agentes, conforme apresentado a seguir.

A primeira questão refere-se à própria estrutura da rede pública de saúde brasileira. Há inúmeras interfaces entre redes hierarquizadas (segundo complexidade dos serviços), redes temáticas (organizadas em torno de agravos, patologias ou fases do clico de vida da população) e redes regionais de atenção à saúde (distribuídas em determinado território), que integram a rede de assistência em saúde do SUS em uma situação de interdependência.

Um serviço de saúde pode, simultaneamente, integrar diferentes redes de atuação do SUS, sendo um fator que influencia no desenvolvimento de cada rede e, em contrapartida, é influenciado pela forma de operacionalização das redes que compõe.

A partir das próprias diretrizes organizativas do SUS, a organização regionalizada das redes de 
serviços de assistência à saúde gera necessidade de interação entre gestores de diversas esferas de governo responsáveis pelos serviços de saúde. A estrutura das diferentes redes de serviços (hierarquizada, temática ou regional) reflete, portanto, decisões tomadas pelos gestores, além de sua própria configuração no passado recente. Consequentemente, a análise global da operacionalização da rede de serviços públicos de saúde, via modelagem com autômatos celulares ou modelagem baseada em agentes, é mais importante para explicar a dinâmica de evolução do SUS do que uma análise estática das partes que compõem o sistema público de saúde.

A segunda questão refere-se à heterogeneidade da rede de serviços de saúde do SUS. A distribuição espacial das ações e serviços de saúde na rede do SUS não é uniforme no território nacional, apresentando importantes variações em quantidade, qualidade e nível de incorporação de tecnologia.

A heterogeneidade dos serviços de saúde prestados no sistema público de saúde brasileiro tem relação direta com as capacidades econômica, fiscal e administrativa das diferentes esferas de governo, ou seja, relaciona-se às características estruturais das unidades locais de governo ${ }^{7}$.

No mesmo sentido, Santos ${ }^{22}$ argumentaria que a heterogeneidade reflete combinações particulares de capital, técnicas e trabalho utilizados em nível local, configurando as denominadas "rugosidades", um dos elementos analíticos da categoria espaço.

Reflexo do passado, as rugosidades atuam como obstáculos ou elementos facilitadores do sistema de saúde no presente. O emprego do conceito de rugosidade na análise espacial das políticas públicas possibilita explicitar lógicas anteriores assumidas pelos serviços de saúde no que diz respeito à distribuição dos serviços, à alocação de profissionais e às prioridades de atendimento, características que influenciam e condicionam novas políticas de saúde ${ }^{23}$.

A análise da rede de serviços do Sistema Único de Saúde a partir de uma perspectiva unidimensional, que desconsidere sua heterogeneidade espacial, pouco informa sobre suas características de evolução (ao longo do tempo) e adaptação às condições da sociedade brasileira. Tampouco esclarece as condições necessárias para a superação de sua lógica intrínseca, intensamente influenciada pelas falhas de mercado identificadas na proteção à saúde da população.

A utilização da modelagem com autômatos celulares ou modelagem baseada em agentes per- mitiria tratar a heterogeneidade na rede de serviços do SUS, caracterizando apropriadamente as sub-redes de diferentes configurações que representam relações entre unidades da federação. Tais sub-redes têm diferentes características, determinadas pelo histórico das políticas de saúde no Brasil e permeada por desigualdades em termos de distribuição de recursos, alocação de profissionais de saúde, prioridades de atendimento e modelos de financiamento.

Os modelos dinâmicos propostos também possibilitam analisar a interação entre diferentes redes do sistema público de saúde (estruturadas a partir da regionalização e hierarquização de serviços de saúde) e ações dos gestores (condicionadas pelo conjunto do regramento infraconstitucional do SUS, condições ambientais e prováveis custos e benefícios).

Por fim, visto que a articulação do modelo de financiamento, princípios e diretrizes organizativas do SUS conduz ao surgimento de falhas de mercado que dificultam a gestão do sistema de saúde, a aplicação de modelos de autômatos celulares ou modelos baseados em agentes possibilitaria uma análise dinâmica de outros mecanismos alternativos apropriados ao financiamento do sistema público de saúde.

Musgrove $^{3}$ ressalta que a desconexão entre orçamento público e avaliação de resultados no setor da saúde, aliada à falta de mecanismos de incentivo ou punição, resultam em políticas públicas de saúde de baixa produtividade em determinados países.

Dado que a ação dos gestores da rede de serviços do SUS segue, entre outras regras, os incentivos dados pelo modelo de financiamento, o uso de AC e ABM permitiria testar o impacto de diferentes regras de financiamento e outros tipos de incentivos na gestão do sistema público de saúde. Permitiria, ainda, analisar a possibilidade de minimizar o impacto das falhas de mercado sobre o sistema público de saúde brasileiro.

Assim, torna-se possível verificar a probabilidade de sucesso ou fracasso dos incentivos propostos no ambiente do sistema de saúde virtual em simulação, antes de tentar a efetiva implementação no SUS. A possibilidade de simulação é uma alternativa que reduz custos ao sistema público de saúde e diminui riscos à saúde da população.

\section{Considerações finais}

Nos últimos vinte anos, inúmeros esforços foram empreendidos à consolidação do Sistema 
Único de Saúde como uma rede descentralizada, hierarquizada e regionalizada, a partir da ênfase na prestação de serviços em âmbito municipal com envolvimento da comunidade atendida e garantia de atendimento integral à saúde do cidadão, sob os princípios e as diretrizes organizativas estabelecidos na concepção de um sistema público de saúde universal.

A evolução do cenário em saúde no Brasil ainda apresenta relativo déficit na oferta de serviços públicos de saúde face às demandas em saúde da população. O redirecionamento das ações do setor público em saúde, a partir do processo de redemocratização ao final da década de 1980, aliado à imprevisibilidade dos problemas de saúde, heterogeneidade dos agentes envolvidos e busca pela universalização da assistência à saúde via construção do SUS, contribui à complexidade intrínseca da rede de políticas públicas de saúde no Brasil, resultando na atual situação de insuficiência no desempenho das funções de governo dentro do âmbito da saúde.

Buscou-se, no presente artigo, demonstrar que os princípios e as diretrizes organizativas do SUS, combinados ao modelo estipulado de financiamento da assistência à saúde, conduzem ao surgimento de falhas de mercado, que geram barreiras à apropriada gestão do sistema e, por conseguinte, impedem a concretização dos objetivos do SUS e, potencialmente, acarretam em aprofundamento das iniquidades em saúde na população.

Adicionalmente, expôs-se a dificuldade analítica gerada pelas características estruturais e heterogeneidade de atores no âmbito da rede de serviços do SUS, tendo em vista as configurações diversas assumidas pelas sub-redes regionalmente definidas, cujos gestores apresentam certo grau de autonomia decisória e mantêm interações entre si segundo regras preestabelecidas.

As características globais do sistema público de saúde brasileiro não são passíveis de compreensão em uma análise simplista da soma de seus agentes componentes, à medida que sua configuração global é resultado da evolução das interações ocorridas em seu interior. O desenvolvimento da rede de serviços do SUS é, portanto, um processo dinâmico, adaptativo e transformador que parte dos próprios agentes que o compõem.

Assim, buscou-se evidenciar as possibilidades analíticas provenientes de uma área de conhecimento inovadora, ainda em desenvolvimento no Brasil: o estudo de sistemas complexos.

Tendo em vista as tecnologias computacionais estabelecidas no país, propõe-se a utilização de métodos quantitativos baseados em dinâmica de agentes inseridos em redes sociais, que incluem redes de políticas públicas, como alternativa adicional ao emprego de métodos quantitativos retrospectivos baseados em estimativas parciais.

A modelagem com autômatos celulares combinada com modelagem baseada em agentes apresentam significativas vantagens à compreensão da organização, heterogeneidade e dinâmica estrutural da rede de serviços do Sistema Único de Saúde, assim como possibilita uma busca pela minimização dos efeitos das falhas de mecanismos de mercado no sistema de saúde brasileiro.

Assim, torna-se possível verificar a probabilidade de sucesso ou fracasso dos incentivos propostos no ambiente do sistema de saúde virtual em simulação, antes de tentar a efetiva implementação no SUS. A possibilidade de simulação é uma alternativa que reduz custos ao sistema público de saúde e diminui riscos à saúde da população.

\section{Colaboradores}

FP Pinheiro Filho participou na concepção, delineamento, redação e aprovação da versão final do artigo; FM Sarti contribuiu na redação, análise, revisão crítica do artigo e aprovação da versão final do artigo. 


\section{Referências}

1. Arrow KJ. Uncertainty and the Welfare Economics of medical care. The Am Econ Rev 1963; 53(5):941973.

2. Jack W. Principles of health economics for developing countries. Washington, DC: The World Bank; 1999.

3. Musgrove P. Health economics in development. Washington, DC: The World Bank; 2004.

4. McPake B, Kumaranayake L, Normand C. Health economics: An international perspective. New York: Routledge; 2002.

5. Brasil. Ministério da Saúde (MS). Sistema Único de Saúde (SUS): Princípios e conquistas. Brasília: MS; 2000.

6. Brasil. Ministério da Saúde (MS). O SUS de A a Z: Garantindo saúde nos municípios. 3a Edição. Brasília: MS; 2009.

7. Arretche M. Estado federativo e politicas sociais: Determinantes da descentralização. Rio de Janeiro: Revan, São Paulo: FAPESP; 2000.

8. World Health Organization (WHO). World Health Organization Constitution. Geneva: WHO; 2006.

9. Toro-Palacio LF, Ochoa-Jaramillo FL. Salud: Un sistema complejo adaptativo. Rev Panam Salud Publica 2012; 31(2):161-165.

10. Stiglitz JE. Economics of the public sector. $3^{\text {rd }}$ Edition. New York: W.W. Norton \& Co; 2000.

11. Joumard I, André C, Nicq C. Health care systems: Efficiency and institutions. Washington, DC: OECD Economics Department; 2010. Working paper.

12. Frank R. Behavioural economics and health economics. NBER WP10881. Cambridge: National Bureau of Economic Research; 2004.

13. Lavras CCC. Descentralização, regionalização e estruturação de redes regionais de atenção à saúde no SUS. In: Ibañez N, Elias PEM, Seixas PHD, organizadores. Política e gestão pública em saúde. São Paulo: Hucitec,Cealag; 2011. p. 317-331.

14. Mueller BPM. Informação assimétrica e teoria dos jogos. Brasília: Ministério das Cidades; 2004.

15. Castro JD. Regulação em saúde: análise de conceitos fundamentais. Sociologias 2002; 4(7):122-135.
16. Viana ALD, Silva HP. Economia e saúde. In: Ibañez N, Elias PEM, Seixas PHD, organizadores. Política e gestão pública em saúde. São Paulo: Hucitec,Cealag; 2011. p. 54-74.

17. Teixeira SMF, Ouverney AM. Gestão de redes: A estratégia de regionalização da política de saúde. Rio de Janeiro: Editora FGV; 2007.

18. Misoczky MC. Redes e hierarquias: uma reflexão sobre arranjos de gestão na busca da equidade em saúde. Rev Adm Pública 2003; 37(2):335-354.

19. Zimbres RA. Modelagem baseada em agentes: Uma terceira maneira de se fazer ciência? In: Anais do 30 . Encontro da ANPAD; 2006; Salvador. [acessado 2011 nov 02]. Disponível em: http://www.dr3.com. br/empresa/enanpadMBA2006.pdf.

20. Lima TFM, Faria SD, Soares Filho BS, Carneiro TGS. Modelagem de sistemas baseada em agentes: Alguns conceitos e ferramentas. In: Anais do $14^{\circ}$ Simpósio Brasileiro de Sensoriamento Remoto; 2009; Natal. p. 5279-5286. [acessado 2011 nov 21]. Disponível em: http://marte.dpi.inpe.br/col/dpi.inpe.br/ sbsr\%4080/2008/11.17.15.46/doc/5279-5286.pdf

21. Furtado BA, Van Delden H. Modelagem urbana e regional com autômatos celulares e agentes: panorama teórico, aplicações e política pública. Rio de Janeiro: IPEA; 2011. (Texto para discussão, n. 1576).

22. Santos M. Por uma Geografia Nova. São Paulo: Hucitec; 1978.

23. Bousquat AEM. Espaço e saúde. In: Ibañez N, Elias PEM, Seixas PHD, organizadores. Politica e gestão pública em saúde. São Paulo: Hucitec, Cealag; 2011. p. 243-260.

Artigo apresentado em 30/04/2012

Aprovado em 17/07/1012

Versão final apresentada em 30/08/2012 